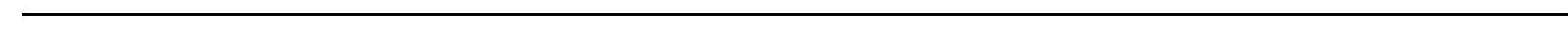

12 


\title{
Using Open Data to Rapidly Benchmark Biomolecular Simulations: Phospholipid Conformational Dynamics
}

\author{
Hanne S. Antila,* Tiago M. Ferreira, O. H. Samuli O llila, and M arkus S. M iettinen* \\ Cite This: J. Chem. Inf. Model. 2021, 61, 938949 \\ Read Online
}

ABSTRACT: Molecular dynamics (MD) simulations are widely used to monitor time-resolved motions of biomacromolecules, although it often remains unknown how closely the conformational dynamics correspond to those occurring in real life. $\mathrm{H}$ ere, we used a large set of open-access M D trajectories of phosphatidylcholine (PC) lipid bilayers to benchmark the conformational dynamics in several contemporary MD models (force elds) against nuclear magnetic resonance (NMR) data available in the literature: e ective correlation times and spin lattice relaxation rates. We found none of the tested MD models to fully reproduce the

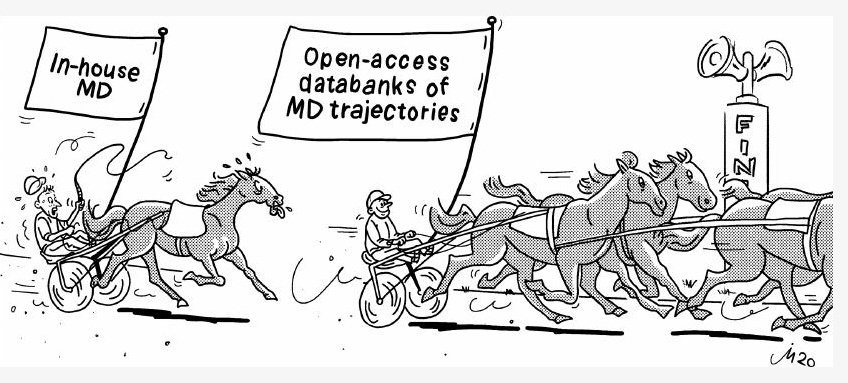
conformational dynamics. That said, the dynamics in CH ARM M 36 and Slipids are more realistic than in the Amber Lipid14, OPLSbased MacRog, and GROM OS-based Berger force elds, whose sampling of the glycerol backbone conformations is too slow. The performance of CH ARM M 36 persists when cholesterol is added to the bilayer, and when the hydration level is reduced. However, for conformational dynamics of the PC headgroup, both with and without cholesterol, Slipids provides the most realistic description because CH ARM M 36 overestimates the relative weight of 1 ns processes in the headgroup dynamics. We stress that not a single new simulation was run for the present work. This demonstrates the worth of open-access MD trajectory databanks for the indispensable step of any serious MD study: benchmarking the available force elds. We believe this proof of principle will inspire other novel applications of MD trajectory databanks and thus aid in developing biomolecular MD simulations into a true computational microscope not only for lipid membranes but for all biomacromolecular systems.

\section{INTRODUCTION}

Ever since the conception of the Protein Data Bank $(P D B)^{1,2}$ and Genbank, ${ }^{3,4}$ open access to standardized and searchable pools of experimental data has revolutionized scienti c research. Constantly growing and improving in delity due to collaborative e ort, ${ }^{5}$ the now hundreds of databanks ${ }^{9}$ fuel the data-driven development of biomolecular structure determination, ${ }^{10}$ re nement, ${ }^{11}$ prediction, ${ }^{12}$ and design ${ }^{13}$ approaches as well as the development of drugs, ${ }_{1}^{14,15}$ materials, ${ }_{1}^{16,17}$ and more. ${ }^{18,19}$ It is clear that open data enables scienti c progress that is far beyond the resources of a single research group or institute. Consequently, the call for public availability and conservation of data has extended to molecular dynamics (MD) simulation trajectories of biomolecules, 20 and the discussion on how and by whom such databanks for dynamic structures would be set up is currently active. ${ }^{23} 26$ While there are currently no general MD databanks in operation, individual databanks are accepting contributions on nucleic acid, ${ }^{27}$ protein/ DNA/RNA, ${ }^{28}$ cyclodextrin, ${ }^{29} \mathrm{G}$-protein-coupled receptor, $^{30}$ and lipid bilayer ${ }^{31}$ simulations.

Since 2013, the NM Rlipids Project (nmrlipids.blogspot. ) has promoted a fully open collaboration approach, where the whole scienti c research process from initial ideas and discussions to analysis methods, data, and publications is all the time publicly available. ${ }^{32}$ While its main focus has been on conformational ensembles of di erent lipid headgroups and on ion binding to lipid membranes, ${ }^{32} 34$ the N M R lipids Project has also built a databank ${ }^{31}$ (zenodo.org/communities/nmrlipids) containing hundreds of atomistic MD trajectories of lipid bilayers and indexed at nmrlipids. .

MD databanks are expected to be particularly relevant for disordered biomolecules, such as biological lipids composing cellular membranes or intrinsically disordered proteins. These, in contrast to folded proteins or DNA strands, cannot be meaningfully described by the coordinates of a single structure alone. Realistic MD simulations, however, can provide the complete conformational ensemble and dynamics of such molecules as well as enable studies of their biological functions in complex biomolecular assemblies. U nfortunately, the current MD force elds largely fail to capture the conformational ensembles of lipid headgroups and disordered proteins. $32,34 \quad 37$ Therefore, before they can be used to draw conclusions, the

Received: N ovember 9, 2020

Published: January 26, 2021 
quality of MD simulations must always be carefully assessed against structurally sensitive experiments. For lipid bilayers, such evaluation is possible against N M R and scattering data. ${ }^{38}$

$\mathrm{H}$ ere, we demonstrate the use of a pre-existing, publicly available set of MD trajectories to rapidly evaluate the delity of phospholipid conformational dynamics in state-of-the-art force elds. The rate at which individual molecules sample their conformational ensemble is traditionally used to assess if a given MD simulation has converged. Going beyond such practicalities, realistic dynamics are particularly desired for the intuitive interpretation of NMR experiments sensitive to molecular motions $s^{39}$ as well as to understand the dynamics of biological processes where molecular deformations play a rate-limiting role, such as membrane fusion. ${ }^{40}$ The here presented comprehensive comparison of dynamics between experiments and di erent MD models at various biologically relevant compositions and conditions is thus likely to facilitate the development of increasingly realistic phospholipid force elds.

Above all, our results demonstrate the power of publicly available MD trajectories in creating new knowledge at a lowered computational cost and high potential for automation. We believe that this paves the way for novel applications of M D trajectory databanks as well as underlines their usefulness not only for lipid membranes but for all biomolecular systems.

\section{METHODS}

Lipid Conformational Dynamics in NMR Data. We analyzed the veracity of phosphatidylcholine (PC) lipid dynamics in $M D$ based on two quantities that are readily available from published ${ }^{39,41}{ }_{43}{ }^{13} \mathrm{C}$ NMR experiments and directly quanti able from atomistic MD simulations: the e ective $\mathrm{C} H$ bond correlation times $\mathrm{e}$ and the spin lattice relaxation rates $R_{1}$.

E ective C HBond Correlation Times e. In alipid bilayer in the liquid crystalline state, each individual lipid samples its internal conformational ensemble and rotates around the membrane normal. Lipid conformational dynamics are re ected in the second-order autocorrelation functions of its $\mathrm{C} \mathrm{H}$ bonds

$$
\left.g()=P_{2}(t) \cdot(t+)\right)
$$

where the angular brackets depict time average, $(t)$ is the unit vector in the direction of the $\mathrm{CH}$ bond at time $\mathrm{t}$, and $\mathrm{P}_{2}$ is the second-order Legendre polynomial $P_{2}(x)=\frac{1}{2}\left(\begin{array}{ll}3 x^{2} & 1\end{array}\right)$.

To analyze the internal dynamics of lipids, the $\mathrm{C} \mathrm{H}$ bond autocorrelation function is often written as a product

$$
g()=g_{f}() g_{s}()
$$

where $g_{\mathrm{f}}()$ characterizes the fast decays owing to, e.g., the internal dynamics and rotation around membrane normal, and $g_{s}()$ the slow decays that originate from, e.g., lipid di usion between lamellae with di erent orientations and periodic motions due to magic-angle spinning conditions (Figure 1). Ferreira et al. ${ }^{41}$ have experimentally demonstrated that, for all phospholipid carbons, the motional correlation times contributing to $g_{f}$ are well below $s$ and to $g_{s}$ well above $100 \mathrm{~s}$. This separation of timescales gives rise to the plateau g( $1 \mathrm{~s} \quad 100$ $\mathrm{s})=\mathrm{S}_{\mathrm{CH}}{ }^{2}$ illustrated in Figure $1 . \mathrm{S}_{\mathrm{CH}}$ is the $\mathrm{C} \mathrm{H}$ bond order parameter

$$
S_{C H}=\frac{1}{2} 3 \cos ^{2}(t) \quad 1
$$
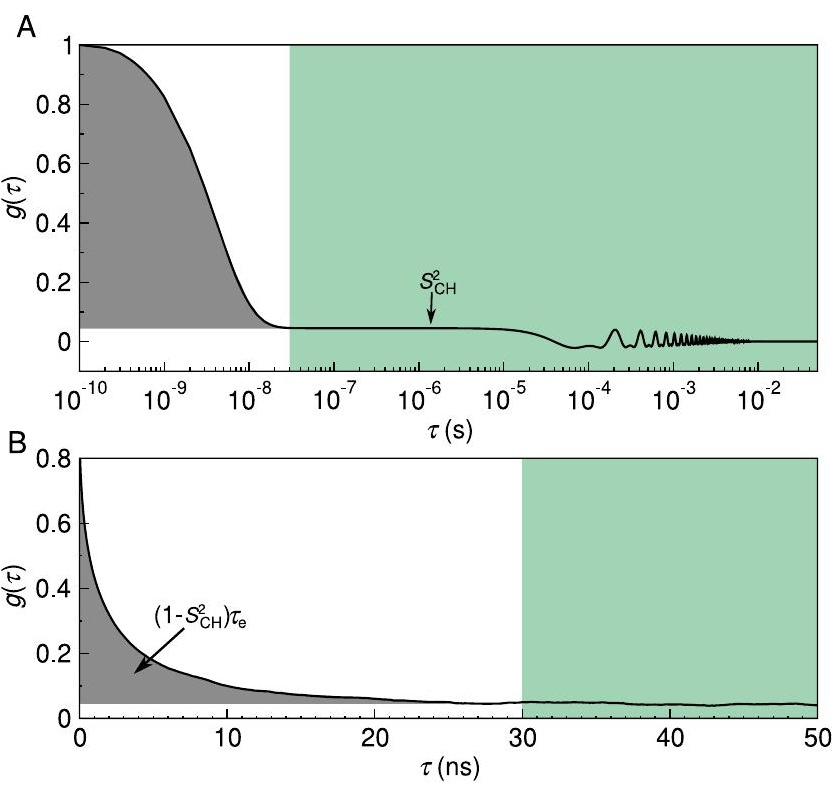

Figure 1. C $\mathrm{H}$ bond autocorrelation function $\mathrm{g}(\mathrm{)}$ ). (A) Idealized illustration of the fast (white background) and the slow (green) mode of the correlation function in solid-state NMR experiments. The fast mode decays to a plateau on which $\mathrm{g}(\mathrm{)})=\mathrm{S}_{\mathrm{CH}}{ }^{2}$, while the slow mode gives the nal descent to zero. 0 scillations at the slow mode region are due to magic-angle spinning. (B) Typical $\mathrm{g}($ ) obtained from an MD simulation, showing the decay toward $\mathrm{S}_{\mathrm{CH}}{ }^{2}$. The gray area under the curve is equal to ( $1 \mathrm{~S}_{\mathrm{CH}}{ }^{2}$ ) $\mathrm{e}$.

where $(\mathrm{t})$ is the angle between the $\mathrm{CH}$ bond and the bilayer normal. $\mathrm{S}_{\mathrm{CH}}$ can be independently measured using dipolar coupling in ${ }^{13} \mathrm{C}$ or quadrupolar coupling in ${ }^{2} \mathrm{H} \mathrm{NMR}$ experiments. Knowing the set of $\mathrm{S}_{\mathrm{CH}}$ for all the $\mathrm{CH}$ bonds in a lipid is highly useful in order to evaluate its conformational ensemble. $^{38}$

As $\mathrm{S}_{\mathrm{CH}}$ describe the conformational ensemble of the lipid, the fast-decaying component $g_{f}$ of the $\mathrm{C} \mathrm{H}$ bond autocorrelation function intuitively re ects the time needed to sample these conformations. The complex internal dynamics containing multiple timescales can be conveniently summarized using the e ective correlation time

$$
e=\frac{g_{\mathrm{f}}() S_{\mathrm{CH}^{2}}{ }^{2}}{1 S_{\mathrm{CH}}{ }^{2}} d
$$

which is related to the gray shaded area below the correlation function in Figure 1. The ${ }_{e}$ detects essentially an average over all the timescales relevant for the lipid conformational dynamics. Their relation to process speeds is intuitive: an increase in longlived correlations increases e.

Spin Lattice Relaxation Rates $\mathrm{R}_{1}$. The $\mathrm{C} \mathrm{H}$ bond dynamics relate to $R_{1}$, the spin lattice relaxation rate, through

$$
R_{1}=\frac{d_{C H}{ }^{2} N_{H}}{20}\left[j\left(H_{C} \quad c\right)+3 j(\quad c)+6 j\left(H^{+} \quad c\right)\right]
$$

where ${ }_{H}$ is the ${ }^{1} \mathrm{H}$ and ${ }_{C}$ the ${ }^{13} \mathrm{CNMR}$ Larmor frequency and $\mathrm{N}_{H}$ is the number of hydrogens covalently bonded to the carbon. The rigid dipolar coupling constant $\mathrm{d}_{\mathrm{CH}} \quad 2 \times 22 \mathrm{kHz}$ for the methylene bond. The spectral density $\mathrm{j}(\mathrm{l})$ is given by the Fourier transformation 

Table 2. Analyzed Open-Access MD Trajectories of Cholesterol-Containing POPC Bilayers at Full Hydration

\begin{tabular}{|c|c|c|c|c|c|c|c|}
\hline force- eld POPC/ water+cholesterol & $c_{\text {chol }}{ }^{a}$ & $\mathrm{~N}_{\text {chol }} \mathrm{b}^{\mathrm{b}}$ & $\mathrm{N}_{1}^{\mathrm{c}}$ & $\mathrm{N}_{\mathrm{w}}{ }^{\mathrm{d}}$ & $\mathrm{T}^{\mathrm{e}}(\mathrm{K})$ & $\mathrm{t}_{\text {anal }}{ }^{f}(\mathrm{~ns})$ & $\operatorname{les}^{9}$ \\
\hline Berger-POPC $-07^{47} / \mathrm{SPC}^{48}$ & $0 \%$ & 0 & 128 & 7290 & 298 & 50 & [63] \\
\hline +H oltje-CH O L-13 ${ }^{64,65}$ & $50 \%$ & 64 & 64 & 10,314 & 298 & 50 & [66] \\
\hline CHARM M $36^{50} / T_{\text {IP } 3 P^{51}}$ & $0 \%$ & 0 & 200 & 9000 & 310 & 500 & [67] \\
\hline+ CHARM M $36^{68}$ & $50 \%$ & 200 & 200 & 18,000 & 310 & 500 & [6 \\
\hline $\mathrm{MacRog} 53 / \mathrm{TIP} \mathrm{P}^{54}$ & $0 \%$ & 0 & 128 & 6400 & 310 & 500 & [70] \\
\hline+ M acRog $^{53}$ & $50 \%$ & 64 & 64 & 6400 & 310 & 500 & [70] \\
\hline 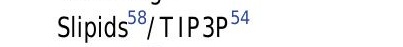 & $0 \%$ & 0 & 200 & 9000 & 310 & 500 & \\
\hline+ Slipids $^{71}$ & $50 \%$ & 200 & 200 & 18,000 & 310 & 500 & [59] \\
\hline
\end{tabular}

${ }^{a}$ Bilayer cholesterol content ( $\left.\mathrm{mol} \%\right) .{ }^{b} \mathrm{~N}$ umber of cholesterol molecules. ${ }^{\mathrm{C}} \mathrm{N}$ umber of POPC molecules. ${ }^{\mathrm{d}} \mathrm{N}$ umber of water molecules. ${ }^{\mathrm{e}} \mathrm{Simulation}$ temperature. ${ }^{\mathrm{f}}$ T rajectory length used for analysis. ${ }^{\mathrm{g}}$ Reference for the openly available simulation les.

Table 3. Analyzed Open-Access MD Trajectories of PC Lipid Bilayers under Varying Hydration Leve

\begin{tabular}{|c|c|c|c|c|c|c|c|}
\hline force eld lipid/ water & lipid & $\mathrm{n}_{\mathrm{w} / \mathrm{I}^{\mathrm{a}}}$ & $\mathrm{N}_{1}^{\mathrm{b}}$ & $\mathrm{N}_{\mathrm{w}}{ }^{\mathrm{c}}$ & $T^{d}(K)$ & $\mathrm{t}_{\text {anal }}{ }^{\mathrm{e}}(\mathrm{ns})$ & les ${ }^{f}$ \\
\hline \multirow[t]{2}{*}{ Berger-POPC $-07^{47} / \mathrm{SPC} 48$} & POPC & 40 & 256 & 10,240 & 300 & 300 & [49] \\
\hline & POPC & 7 & 128 & 896 & 298 & 60 & [72] \\
\hline \multirow[t]{4}{*}{ Berger-DLPC $-13^{73} / S P C-E^{61}$} & DLPC ${ }^{g}$ & 24 & 72 & 1728 & 300 & 80 & [74] \\
\hline & DLPC ${ }^{g}$ & 16 & 72 & 1152 & 300 & 80 & [75] \\
\hline & DLPC ${ }^{g}$ & 12 & 72 & 864 & 300 & 80 & [76] \\
\hline & DLPC ${ }^{g}$ & 4 & 72 & 288 & 300 & 80 & [77] \\
\hline \multirow[t]{5}{*}{ CHARM M $36^{50} /{\text { TIP } 3 P^{51}}^{2}$} & POPC & 40 & 128 & 5120 & 303 & 140 & [78] \\
\hline & POPC & 34 & 256 & 8704 & 300 & 500 & [55] \\
\hline & POPC & 31 & 72 & 2232 & 303 & 20 & [79] \\
\hline & POPC & 15 & 72 & 1080 & 303 & 20 & [80] \\
\hline & POPC & 7 & 72 & 504 & 303 & 20 & [81] \\
\hline \multirow[t]{5}{*}{ M acRog ${ }^{53} /$ TIP $\mathrm{P}^{54}$} & POPC & 50 & 288 & 14,400 & 310 & 40 & [82] \\
\hline & POPC & 25 & 288 & 7200 & 310 & 50 & [82] \\
\hline & POPC & 15 & 288 & 4320 & 310 & 50 & [82] \\
\hline & POPC & 10 & 288 & 2880 & 310 & 50 & [82] \\
\hline & POPC & 5 & 288 & 1440 & 310 & 50 & [82] \\
\hline
\end{tabular}

${ }^{a}$ Water/ lipid molar ratio. ${ }^{b} \mathrm{~N}$ umber of lipid molecules. ${ }^{c} \mathrm{~N}$ umber of water molecules. ${ }^{\mathrm{d}}$ Simulation temperature. ${ }^{\mathrm{T}}$ rajectory length used for analysis. ${ }^{f}$ Reference for the openly available simulation les. ${ }^{91,2-D i l a u r o y l-s n-g l y c e r o-3-p h o s p h o c h o l i n e . ~}$

the whole trajectory and then obtaining the error estimate

$g_{f}()$ as the standard error of the mean over the $N_{\text {, lipids. }}$ Importantly, this gives an uncertainty estimate for $g_{f}(\quad)$ at each time point .

To obtain the lower bound on e, we integrate the function $g_{\mathrm{f}}() \quad g_{\mathrm{f}}()$ over time from $=0$ until $=\mathrm{t}_{\mathrm{t}}$. Here

$$
t_{1}=\min \left\{\left\{t \mid g_{f}(t) \quad g_{f}(t)=0\right\}, \frac{t_{\text {anal }}}{2}\right\}
$$

That is, $t_{1}$ equals the rst time point at which the lower error estimate of $g_{f}$ reached zero, or $t_{1}=t_{\text {anal }} / 2$, if zero was not reached before that point.

To obtain the upper error estimate on e, we rst integrate the function $g_{f}()+g_{f}()$ over time from $=0$ until $t_{u}=\min \left\{t_{0}\right.$, $\left.t_{\text {anal }} / 2\right\}$. Note, however, that this is not yet su cient, because there could be slow processes that the simulation was not able to see. Although these would contribute to e with a low weight, their contribution over long times could still add up to a sizable e ect on e. That said, it is feasible to assume (see Figure 1A) that there are no longer-time contributions to $\mathrm{g}_{\mathrm{f}}$ than something that decays with a time constant of $10^{6} \mathrm{~s}$. We use this as our worst case estimate to assess the upper bound for e, that is, we assume that all the decay of $g_{f}$ from the time point $t_{u}$ onward comes solely from this hypothetical slowest process that decays with a time constant of $10^{6} \mathrm{~s}$. The additional contribution to the upper bound for ethen reads

$$
\begin{aligned}
& \left.\left(g_{f}\left(t_{u}\right)+g_{f}\left(t_{u}\right)\right) \quad{ }^{10} s \exp \frac{t_{u}}{10^{6} s}\right)_{\lambda} d
\end{aligned}
$$

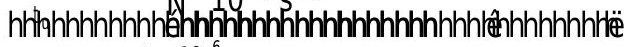

$$
\begin{aligned}
& =\left(1 \exp \left(\frac{t_{u} 10^{6} \mathrm{~s}}{10{ }^{6} \mathrm{~s}}\right)\right) 10^{6} \mathrm{~s}
\end{aligned}
$$

The $R_{1}$ rates were calculated using eq 5 . The spectral density $j\left(\right.$ ) was obtained from the normalized correlation function $g_{f}$ by tting it with a sum of 61 exponentials

$$
g_{f}()_{i=1}^{61} e^{/ i}
$$

with logarithmically spaced timescales i ranging from 1 ps to 1 $s$ and then calculating the spectral density of this $t$ based on the Fourier transformation ${ }^{41}$

$$
j()=2\left(\begin{array}{ll}
1 & S_{C H}
\end{array}\right)_{i=1}^{61} i \frac{i}{1+i}
$$

The $R_{1}$ rate of a given $C H$ pair was rst calculated separately for each lipid $m$ ( using eq 5 with $N_{H}=1$, and $j{ }^{m}($ ) obtained for the normalized correlation function $\left.g_{f}{ }^{m}\right)$. The resulting $N_{1}$ measurements per $\mathrm{C} \mathrm{H}$ pair were then assumed independent: their mean gave the $\mathrm{R}_{1}$ rate of the $\mathrm{CH}$ pair, and the standard error of the mean its uncertainty. The total $R_{1}$ rate of a given carbon was obtained as a sum of the $R_{1}$ rates of its $C H$ pairs. When several carbons contribute to a single experimental $R_{1}$ rate 


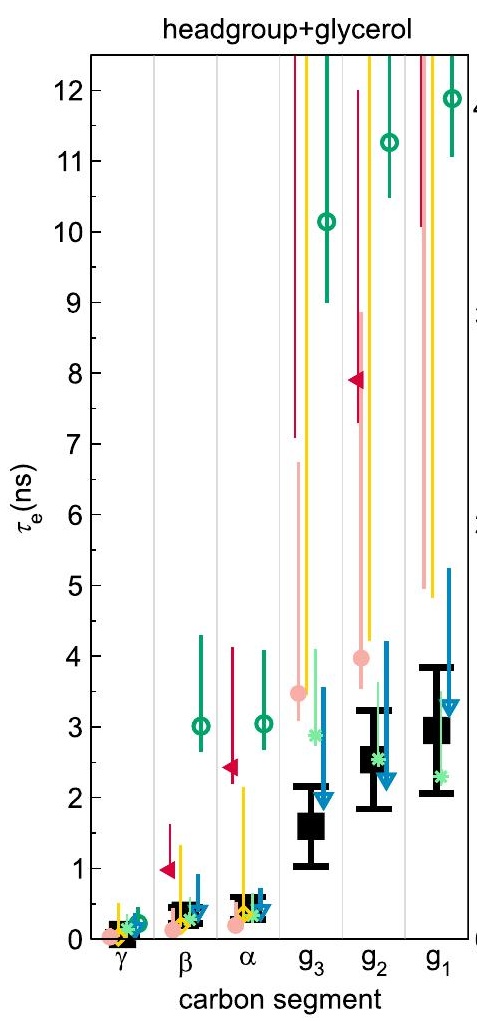

$2^{\prime} \quad 3^{\prime}$

$14^{\prime} 15^{\prime} 16^{\prime}$
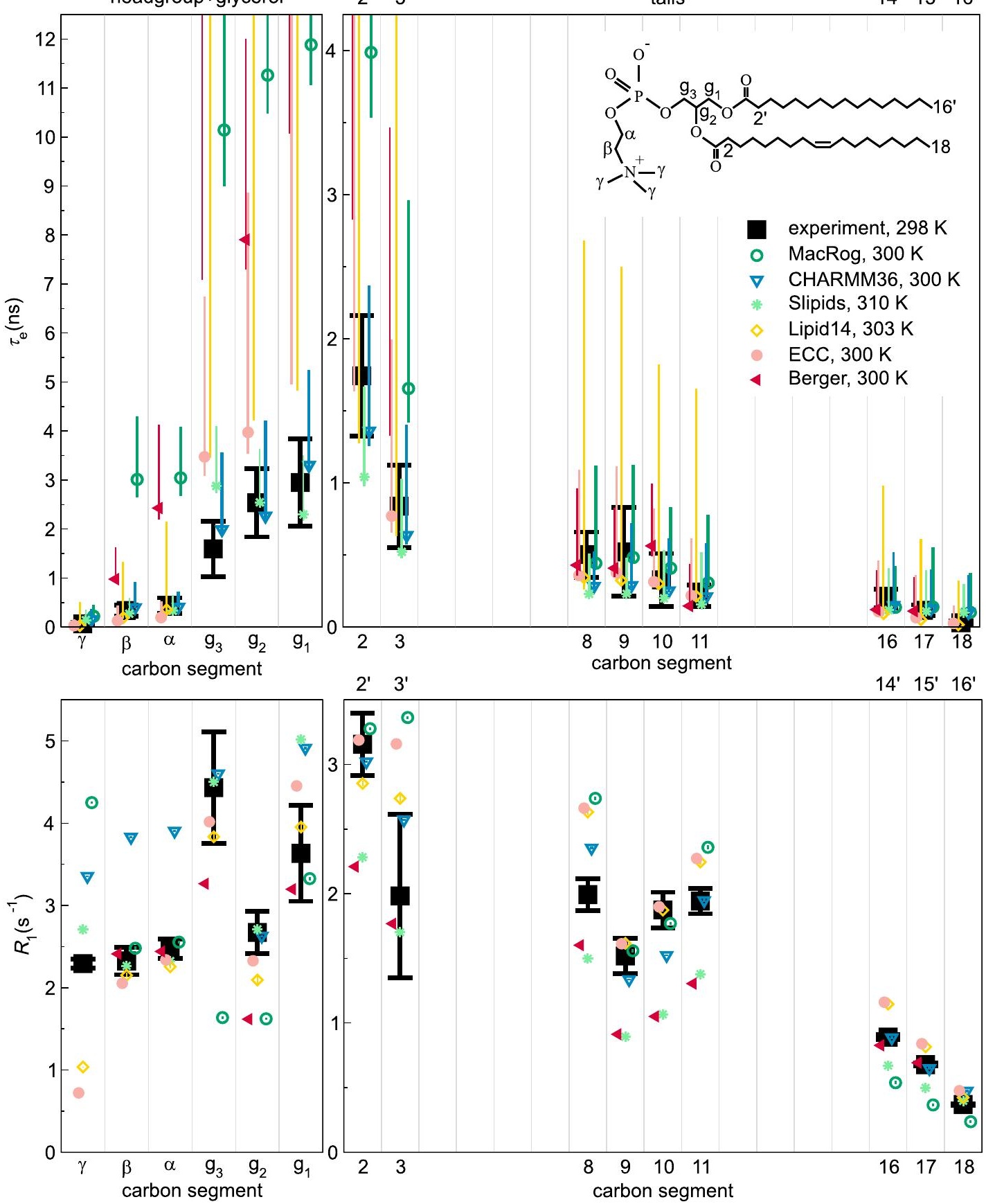

$2^{\prime} \quad 3^{\prime}$

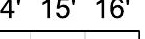

Figure3. E ective correlation times ( $e_{e}$ top) and $R_{1}$ rates (bottom) in experiments ${ }^{39}$ (black) and MD simulations (colored) of POPC bilayers in the $L$ phase under full hydration. Inset shows the POPC chemical structure and carbon segment labeling. Each plotted value contains contributions from all the hydrogens within its carbon segment; the data for segments 811 are only from the sn-2 (oleoyl) chain, whereas the (experimentally nonresolved) contributions of both tails are included for segments $23(2 \quad 3$ in the sn-1 chain) and $16 \quad 18$ (14 16 ). Simulation results are only shown for the segments for which experimental data were available. For e, a simulation data point indicates the average over $\mathrm{C} H$ bonds; however, if ${ }_{e}$ could not be determined for all bonds, only the error bar (extending from the mean of the lower to the mean of the upper error estimates) is shown. The Berger data for segments , C18, and C16 are left out as the protonation algorithm used to construct the hydrogens post-simulation in united atom models does not preserve the methyl $\mathrm{C} \mathrm{H}$ bond dynamics. Table 1 provides further simulation details, while information on the experiments is available at ref 39 .

due to the overlapping peaks (for example, in C2 carbon in the acyl chains and the carbons), the $R_{1}$ from simulations was obtained as an average over carbons with overlapping peaks. The segment-wise error estimates were obtained by standard error propagation, starting from the uncertainties of the $R_{1}$ rates of the C $\mathrm{H}$ pairs.
To gain some qualitative insight on the timescales at which the main contributions to the $R_{1}$ rates arise, we also calculated "cumulative" $R_{1}$ rates, $R_{1}($ ), which contained those terms of the sum in eq 12 for which $i<$. N ote that here the $g_{f}$ averaged over lipids was used; therefore, the "cumulative" $R_{1}(\quad$ ) does not necessarily have exactly the same numerical value as the actual $R_{1}$. 
Finally, we note that the $t$ of eq 11 provides an alternative to estimating e because

$$
e^{=} g_{f}() d \quad i i
$$

When the simulation trajectory is not long enough for the correlation function to reach the plateau, integrating $g_{f}$ gives a lower bound estimate for e, while the sum of eq 13 includes also (some) contribution from the longer-time components via the tting process. However, in practice, the $t$ is often highly unreliable in depicting the long tails of the correlation function, and thus we chose to quantify $e$ using the area under $g_{f}$ and estimate its uncertainty as detailed above.

\section{RESULTS AND DISCUSSION}

Using open-access MD simulation trajectories, we benchmark phospholipid conformational dynamics in six MD force elds. We start with pure PO PC bilayers in their liquid crystalline fully hydrated state ( see T able 1 for simulation details and Figures 3 and 4 for the data) and then proceed to check the changes in dynamics when cholesterol is added to the bilayer ( $T$ able 2 and Figure 5) and when the hydration level is reduced (T able 3 and Figure 6). 0 ur yardsticks are the e ective correlations times (eq 4) and the $\mathrm{R}_{1}$ rates (eq 5) measured at $125 \mathrm{M} \mathrm{Hz}^{13} \mathrm{C}(500$ $\mathrm{MHz}{ }^{1} \mathrm{H}$ ) Larmor frequency; an $\mathrm{MD}$ model with correct rotational dynamics in a window around $1 \mathrm{~ns}$ will match the experimental $R_{1}$ rates, whereas the e re ect all the sub- $s$ timescales ( Figure 1).

Pure POPC at Full Hydration: Slipids and CHARMM 36 Reproduce e Excellently. The top panels of Figure 3 compare the e ective correlation times e obtained for fully hydrated POPC bilayers in experiments (black) and in six di erentMD force elds (color). We seethat as implied by the discussion leading to eq 10 sub- SMD simulations typically lead to asymmetric error bars on $e ;$ if these open-access trajectories were extended, the e values would more likely increase than decrease. Qualitatively, every force eld captures the general shape of the e pro le: dynamics slows down toward the glycerol backbone in both the headgroup and the tails.

Q uantitatively, most MD simulations tend to produce too slow dynamics in the glycerol region (Figure 3). This is consistent with previous results for the Berger model ${ }^{41}$ and with the insu cient conformational sampling of glycerol backbone torsions observed in 500-ns-long CHARM M c $32 \mathrm{b2}^{87,88}$ simulations of a PC lipid. ${ }^{89}$

The best overall e performance is seen in Slipids and in particular CHARMM 36 (Figure 3). This is in line with CHARM M 36 reproducing the most realistic conformational ensembles for the headgroup and glycerol backbone among the MD simulation force elds benchmarked here. ${ }^{32,34}$ Indeed, it is important to keep in mind that the conformational ensembles greatly di er between force elds and are not exactly correct in any of them. ${ }^{32,34}$ Consequently, the calculated e times and $R_{1}$ rates depict the dynamics of sampling a somewhat di erent and incorrect phase space for each model. To this end, we try to avoid overly detailed discussion on the models and rather concentrate on common and qualitative trends. That said, there are a few carbon segments in the data for which the experimental order parameters, $R_{1}$, and e are all (almost) reproduced by simulations, suggesting that both the conformational ensemble and the dynamics are correctly captured by MD in these cases. For example, Slipids performs well at the and and
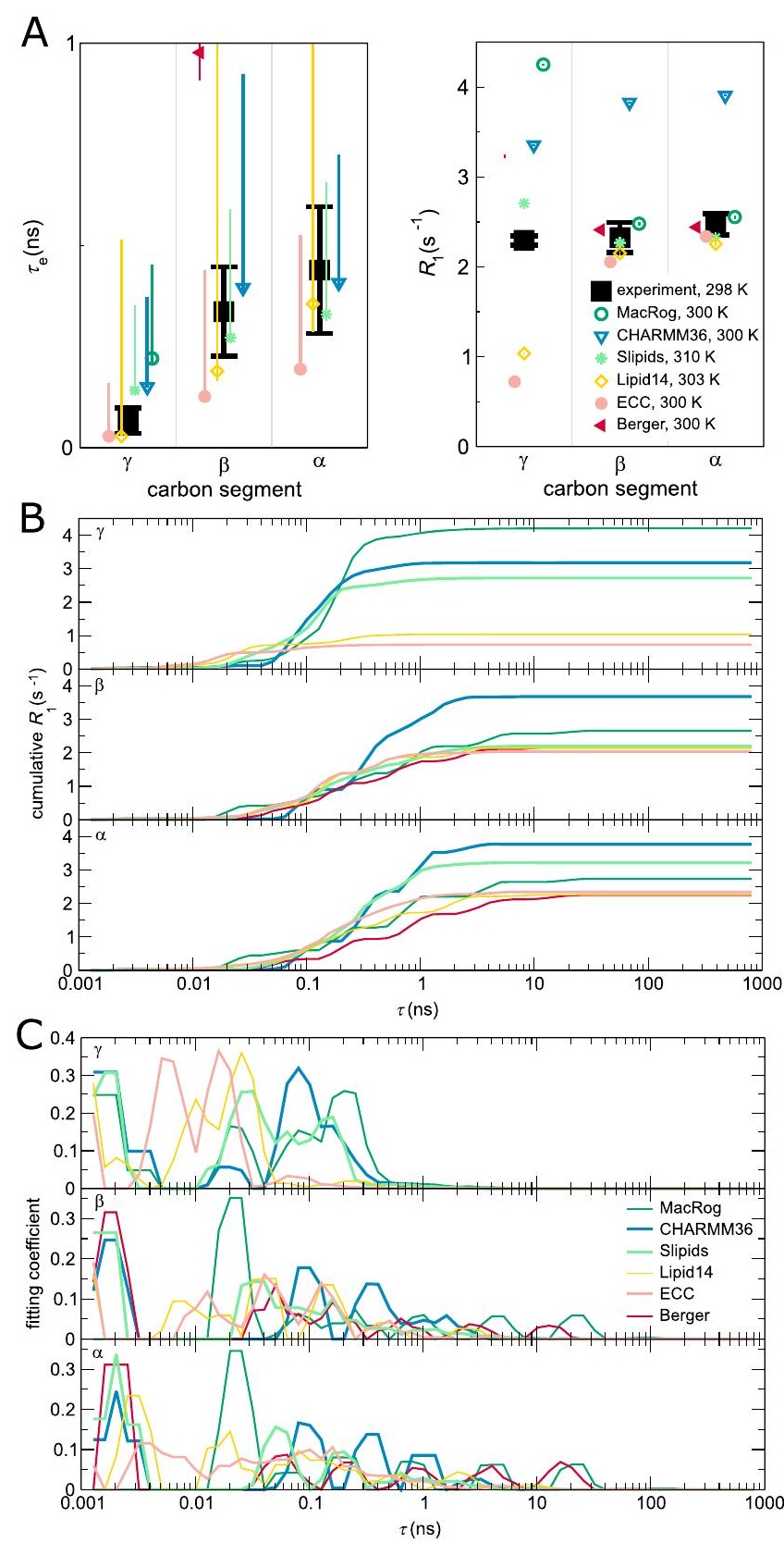

Figure 4. Contributions to the dynamics of the headgroup segments. (A) Zoom on the headgroup e (left panel) and $R_{1}$ (right). (B) "Cumulative" $R_{1}$ ( ) of the (top panel), (middle), and (bottom) segments. $R_{1}($ ) is obtained, as detailed in M ethods, by including in the sum of eq 12 only terms with $<$. Consequently, at , the $R_{1}($ ) approaches the actual $R_{1}$. (C) Prefactor weights from eq 11 of (top),

(middle), and (bottom). N ote that panels (B) and (C) show a sliding average over three neighboring data points.

CHARMM 36 at the $g_{3}, g_{2}$, and C2 segments. These are, however, exceptions.

An Excellent e May Be Accompanied by a Poor $\mathrm{R}_{1}$, or Vice Versa. The lower panels of Figure 3 compare the experimental and simulated $R_{1}$ rates under the same conditions that were used for the e above. Notably, there are several instances where the $R_{1}$ comparison distinctly di ers from what was seen for $\mathrm{e}$.

There are cases where a matching $R_{1}$ is accompanied by a larger-than-experimental e. MacRog for the , , and $g_{1}$ segments provides a prominent example of this. Such a 





